Rev. Hist., $\mathrm{N}^{\circ} 27$, vol. 1, Enero-Junio 2020: 25-44

ISSN 0717-8832

\title{
Democracia y Desarrollo. Una aproximación a la Alianza para el Progreso en Venezuela, 1961-1969
}

Democracy and Development. An approach to the Alliance for Progress in Venezuela, 1961-1969

Froilán Ramos Rodríguez*

\section{RESUMEN}

Este trabajo analiza la recepción e influencia de la Alianza para el Progreso en Venezuela durante los años sesenta (1961-1969). El programa de asistencia estadounidense financió distintos proyectos sociales en países de América Latina, con el objeto de promover el desarrollo local, y a la vez contrarrestar el impacto de la Revolución Cubana (1959). Metodológicamente, la investigación se nutrió de fuentes primarias, documentos oficiales venezolanos y estadounidenses, y revisión de prensa contemporánea. En suma, se evidencia que si bien los recursos económicos enviados a Venezuela fueron inferiores a otras naciones, el respaldo político de Estados Unidos fue fundamental para la consolidación de la joven democracia venezolana.

Palabras claves: Alianza para el Progreso, Desarrollo, Democracia, Venezuela, Estados Unidos, Guerra Fría

\section{ABSTRACT}

This paper analyzes the reception and influence of the Alliance for Progress in Venezuela during the 1960s (1961-1969). The US assistance program financed various social projects in Latin American countries, with the aim of promoting local development, and at the same time counteracting the impact of the Cuban Revolution (1959). Methodologically, the research was nurtured from primary sources, official Venezuelan and American documents, and contemporary press review. In sum, it is evident that although the economic resources sent to Venezuela were inferior to other nations, the political support of the United States was fundamental for the consolidation of the young Venezuelan democracy.

Keywords: Alliance for Progress, Development, Democracy, Venezuela, United States, Cold War

Recibido: Julio 2019

Aceptado: Octubre 2019

"Todos los hombres, en algún momento de su vida, se sienten solos; y más: todos los hombres están solos".

Octavio Paz

(1972. El laberinto de la soledad, México, D.F., Fondo de Cultura Económica, p. 175).

\footnotetext{
* Doctor en Historia. Universidad Católica de la Santísima Concepción, Chile. Email: framos@ucsc.cl
} 


\section{Introducción}

Al rememorar las palabras de Octavio Paz, pareciera que todos los hombres alrededor de la Alianza para el Progreso se fueron quedando solos, solos en sus ilusiones y expectativas. A la luz del tiempo, todo el decenio de los sesenta, marcado por las ideas de Alianza y sus protagonistas, sigue despertando atracción bien por lo notable de sus planteamientos o por lo utópico de su práctica.

El 13 de marzo de 2019, se cumplieron cincuenta y ocho años del anuncio de la Alianza para el Progreso, por parte del presidente John F. Kennedy de los Estados Unidos. Luego de más de cinco décadas de su presentación y de dos del término de la Guerra Fría (1991), la Alianza amerita una revisión desapasionada y crítica de su origen, marcha y repercusiones en Venezuela.

Desde un momento temprano, Venezuela se convirtió en uno de los socios y/o aliados principales para el presidente Kennedy y la Alianza para el Progreso en la región. La cercanía en el pensamiento reformador del socialdemócrata Rómulo Betancourt y el mandatario estadounidense, sumado a los valores conjuntos en torno a la democracia y la libertad en el continente, habían consagrado un ápice clave en la proyección de la Alianza en el país suramericano y en la región.

La Alianza para el Progreso ha sido un tema atractivo para los historiadores contemporáneos, quienes han reflejado este interés en una importante producción. Dentro de ellos, destacan los trabajos de Stephen Rabe (1999), Thomas Wright (2001), Jeffrey Taffet (2007), y más recientemente el de Aragorn Storm Miller (2016), entre otros, quienes aportan distintas perspectivas sobre la Alianza, desde la influencia de las relaciones internacionales (Rabe), los movimientos armados de izquierda en la región (Wright), la comparación de casos (Taffet), y la coyuntura externa e interna (Miller).

En este sentido, tres preguntas centrales guían este trabajo: ¿Cómo fue recibida la Alianza en Venezuela? ¿Cuál fue su desarrollo ejecutivo en el país? y ¿Qué repercusión tuvo? Para contestar estas interrogantes, se recurre al análisis de fuentes primarias, principalmente documentación oficial impresa y el apoyo en prensa de la época, que permite seguir con mayor detalle el contexto de los sesentas.

\section{Ideas desarrollistas previas a la Alianza para el Progreso}

A comienzos del siglo XX, Venezuela experimentó la larga dictadura del general Juan Vicente Gómez. Los veintisiete años de régimen militar (1908-1935) significaron un letargo político, y lento pero sostenido proceso de transición económica; de una Venezuela agroexportadora de café a una Venezuela rentista de petróleo, conservando las condiciones de dependencia y monoproducción. 
Para el escritor Mariano Picón Salas, Venezuela entró al siglo XX en 1936, con más de tres décadas de retraso. Ciertamente, fue entre 1936 y 1945 que se produjo una transición política hacia formas con visos democráticos y una mayor participación en las ganancias producidas por el petróleo ${ }^{1}$. Gracias en parte a la coyuntura de la Segunda Guerra Mundial, el Estado venezolano pudo disponer de una Ley de Impuesto sobre la Renta en 1942 y una Ley de Hidrocarburos en 1943, que le permitieron obtener mayores dividendos de la extracción de petróleo por las compañías extranjeras (como la Standard Oil).

Desde 1945, Venezuela había comenzado a experimentar una serie de rápidos cambios políticos y económicos, auspiciados por la Junta Revolucionaria de Gobierno (1945-1948), presidida por Rómulo Betancourt y conformada por dirigentes civiles del partido socialdemócrata Acción Democrática (AD) y por militares. La Junta aspiraba a implementar reformas políticas para la democratización del país, y reformas económicas para la industrialización del aparato productivo.

En este sentido, dentro del equipo económico con ideas desarrollistas se encontraban el ingeniero Eduardo Mendoza, ministro de Agricultura y Cría, el doctor Juan Pablo Pérez Alfonzo, ministro de Fomento, y el economista Manuel Pérez Guerrero, ministro de Hacienda. En este período, se creó la Corporación Venezolana de Fomento en 1946, como institución promotora del Estado venezolano para el financiamiento de proyectos de industrialización, electrificación y sustitución de importaciones. A pesar del golpe de estado de 1948, y el establecimiento de la dictadura militar de Marcos Pérez Jiménez, los planes desarrollistas continuaron y se potenciaron con la creación de la Oficina de Estudios Especiales (OEE) en 1953, a cargo del coronel (luego general) Luis Felipe Llovera Páez. La OEE fue la responsable de la planificación de industrias básicas como las empresas siderúrgicas en la región de Guayana (zona de la selva amazónica), al sur de Venezuela².

Los años cincuenta fueron esenciales en esa concepción en torno al desarrollo en Venezuela. Por un lado, el gobierno de Pérez Jiménez se convirtió en la primera dictadura militar desarrollista de América Latina, al promover grandes proyectos de infraestructura, vialidad, industria básica y petroquímica ${ }^{3}$. Mientras que, por otro lado, en la región crecía la influencia

\footnotetext{
1 Puede verse: Ramos Rodríguez, Froilán. 2013. “Venezuela 1936-1945. ¿Democracia bajo tutelaje militar o transición pretoriana?", pp. 101-131. en Cardozo, Alejandro. y Luis Buttó (Directores). El Incesto Republicano. Relaciones Civiles y Militares en Venezuela 1812-2012, Caracas, Nuevos Aires.

2 Véase: Guzmán, Guillermo. 2009. "La Oficina de Estudios Especiales de la Presidencia de la República: la planificación de la Industria Siderúrgica Nacional y el desarrollo de Guayana (1953-1958)", Tiempo y Espacio, Vol. 19, No. 52, Caracas, pp. 251-268.

3 Véase Ramos Rodríguez, Froilán. y G. Guía Caripe, Germán. 2013. "Proyecto de creación una Industria Básica de apoyo a la Fuerza Armada de Venezuela durante el Gobierno militar de Marcos Pérez Jiménez (1952-1958)", RUHM, Vol. 2, No. 3, Madrid, pp. 203-218; Castillo D'Imperio, Ocarina. 2003. Los años del Buldozer: ideología y política, 1948-
} 
del pensamiento desarrollista de la CEPAL, en particular en el economista venezolano José Antonio Mayobre, quien más tarde se convertiría en uno de los principales exponentes regionales de la tendencia cepaliana y promotores de reformas en la administración pública venezolana ${ }^{4}$.

El término de los cincuenta devino en drásticos cambios en el escenario nacional e internacional. En enero de 1958, fue derrocada la dictadura perezjimenista, y meses más tarde, en mayo, el vicepresidente de los Estados Unidos, Richard Nixon, visitó Caracas en medio de un ambiente tenso y exacerbado. Además, el triunfo de la Revolución Cubana en enero 1959, impulsó a los movimientos de izquierda en América Latina a buscar formas de lucha armada.

En este contexto, un nuevo y joven mandatario estadounidense, John F. Kennedy, recurrió a un equipo de académicos norteamericanos, como Richard "Dick" Goodwin, Lincoln Gordon, Robert Alexander, Arthur Whitaker y Adolf Berle, junto con asesores de la CEPAL como el argentino Raúl Prebisch, el chileno Felipe Herrera y el venezolano Mayobre, para delinear lo que sería finalmente la Alianza para el Progreso ${ }^{5}$. Más adelante, el 13 de marzo, en la Casa Blanca, ante los representantes diplomáticos latinoamericanos, el presidente Kennedy anunció formalmente la Alianza para el Progreso:

"si hemos de afrontar un problema de tan imponentes dimensiones, nuestro proceder debe ser audaz y a tono con la concepción majestuosa de la Operación Panamericana. Por eso he hecho un llamamiento a todos los pueblos del hemisferio para que nos unamos en una Alianza para el Progreso, en un vasto esfuerzo de cooperación, sin paralelo en su magnitud y en la nobleza de sus propósitos, a fin de satisfacer las necesidades fundamentales de los pueblos de América, las necesidades fundamentales de techo, trabajo y tierra, salud y escuelas" ${ }^{\prime \prime}$.

Por vez primera, un presidente estadounidense se refería con preocupación por los problemas existentes en América Latina, en un tono de optimismo que trasmitía una expectativa generalizada en el continente, en muy poco tiempo Kennedy había logrado despertar la atención de norteamericanos y latinoamericanos en torno las posibilidades de poder realizar algo diferente y sin precedentes en el hemisferio ${ }^{7}$.

1958, Caracas, Tropykos; López Portillo, Felícitas. 1986. El Perezjimenismo: génesis de las dictaduras desarrollistas, México, DF, UNAM, entre otros.

4 Para profundar el tema, puede consultarPuede consultarse: Mayobre, José. Antonio. 2007. Latinoamérica: Economía y desarrollo, Caracas, Banco Central de Venezuela.

5 Véase Ramos Rodríguez, Froilán. y Javier Castro Arcos, Javier. 2014. "La Alianza para el Progreso en Chile y Venezuela, 1961-1963", Tiempo y Espacio, No. 62, Caracas, pp. 99-138.

${ }^{6}$ Alianza para el Progreso. 1961. Documentos Básicos, Punta del Este, s.e., p. 4

7 Paul Johnson crítica las altas metas de JFK, señalando: "ahí estaba la debilidad del universalismo de Kennedy" (Johnson, P. 2000. Tiempos Modernos, Buenos Aires, Vergara, p. 478.). 


\section{Recepción de la Alianza para el Progreso en Venezuela}

La administración de Rómulo Betancourt (1959-1964) tuvo que hacer frente a difíciles obstáculos para la naciente democracia venezolana, provenientes tanto del exterior como del interior del país, y de tendencias radicales de izquierda y derecha. El 23 de enero de 1958, fue derrocado el gobierno militar del general Marcos Pérez Jiménez, y se inició el proceso de democratización del país, se convoca a elecciones para fines de este año, las cuales fueron ganadas por el candidato del partido Acción Democrática, Rómulo Betancourt. No obstante, esta era todavía una precoz y frágil democracia en el continente americano.

En el plano interno, grupos dentro de las fuerzas armadas conspiraban; por un lado, oficiales de vieja usanza pretoriana, como el general Jesús Castro León en abril de $1960^{8}$; mientras que, por otro lado, organizaciones políticas de izquierda, el Partido Comunista y el Movimiento de Izquierda Revolucionaria (MIR), promovían golpes de estado, impulsados ideológicamente por la Revolución Cubana9.

Internacionalmente, el gobierno dictatorial de Rafael Trujillo en la República Dominicana promovía la desestabilización del gobierno democrático, y atentados contra la vida del presidente Betancourt, como el del 24 de junio de $1960^{10}$, durante el desfile por el Día del Ejército, del cual resultó ileso; mientras que Fidel Castro desde Cuba, promovía con dinero y logística la violencia de los partidos de izquierda, financiando guerrillas rurales y terrorismo urbano $^{11}$.

Por su parte, la política exterior de la Administración Betancourt estaba dirigida por los principios de integración latinoamericana, cooperación hemisférica y la "Doctrina Betancourt", la cual promovía el sistema democrático en el continente en contra de cualquier usurpación del poder, contra golpes de estados, y el no reconocimiento de gobiernos militares ni comunistas ${ }^{12}$. Betancourt se convirtió en el principal líder latinoamericano defensor de la democracia en la región ${ }^{13}$, tanto contra las dictaduras de derecha como de las de izquierda.

\footnotetext{
8 Irwin Gáffaro, Domingo. 2006. Control Civil y Pretorianismo en Venezuela, Caracas, Universidad Católica Andrés Bello, p. 170.

${ }^{9}$ Buttó, Luis.Alberto. 2015. "El golpismo se viste de blanco. Insurrecciones navales contra la naciente democracia venezolana", Tiempo y Espacio, No. 64, Caracas, pp. 509-544.

${ }^{10}$ Durante este incidente, Betancourt resultó herido, mientras que el jefe de la Casa Militar, el coronel Ramón Armas Pérez, falleció. Betancourt, B. 1962. Tres años de gobierno democrático, 1959-1962, Caracas, Imprenta Nacional, p. 318.

11 Tarver, H. Michael. 2001. Venezuelan Insurgency, 1960-1968: A successful failure, Bloomington, Indiana, Xlibris, pp. 87-89.

12 Romero, María Teresa. 2005. Venezuela en defensa de la democracia, 1958-1998. El caso de la Doctrina Betancourt. Caracas, Fondo para la Cultura Urbana, p. 25.

13 "Venezuela's President Betancourt", Times (New York), Feb. 8, 1960.
} 
El presidente John F. Kennedy fue el primer mandatario estadounidense en visitar Venezuela. Su llegada a la nación y su permanencia en Caracas y La Morita (estado Aragua) fue ampliamente seguida por los medios nacionales. La imagen del joven presidente Kennedy fue recogida con expectación y entusiasmo en la prensa venezolana.

De este modo, aparecieron titulares que recogían la atmosfera de la nación, el diario El Nacional publicó: “Kennedy huésped de Venezuela. Invocó el espíritu de Bolívar y Roosevelt" ${ }^{14}$; mientras El Universal escribió: "Entusiasta recibimiento tributó al presidente Kennedy y su esposa el Pueblo de Venezuela", a la vez que agregó las primeras palabras de Kennedy al llegar al aeropuerto: "Vengo siguiendo los pasos de Franklin Delano Roosevelt, porque fue él quien mejor entendió la independencia de nuestros dos países"15. Incluso, uno de los diarios críticos del gobierno Betancourt, como Últimas Noticias, plasmó aquel momento: "Kennedy y Jacqueline recibidos con grandes demostraciones de aprecio" ${ }^{16}$.

En general, el ambiente en la opinión pública trasmitía optimismo y ánimo en las relaciones venezolano-estadounidenses. Así, por ejemplo, antes de la visita de Kennedy, una comisión del senado de Estados Unidos arribó a Venezuela. Estaba integrada por John L. MacLellam, demócrata por Arkansas, Michael Mansfied, Alan Bible, Margaret Chase Smith, Henry Dworshak y Roman Lee Hruska, al llegar a la nación declararon: "Venezuela es el país guía en Latinoamérica del Plan 'Alianza para el Progreso'”17. En este mismo orden, el diario caraqueño La Esfera expresaba:

"Desde el punto de vista sudamericano nada impresiona más y mejor que el proyecto ideado por el presidente Kennedy de una 'alianza para el progreso' que termine con el subdesarrollo, la miseria y la ignorancia en el continente sudamericano y lo ponga en el mismo grado de progreso y prosperidad que ha alcanzado la nación del Norte" ${ }^{18}$.

La Iglesia católica, por su parte, se mostró optimista con los propósitos del presidente Kennedy y la Alianza. De hecho, la revista católica (jesuita) SIC reprodujo el discurso de toma

\footnotetext{
14 “Kennedy huésped de Venezuela", El Nacional (Caracas), 17 de diciembre de 1961, primera página.

15 “Entusiasta recibimiento tributó al presidente Kennedy y su esposa el Pueblo de Venezuela”, El Universal (Caracas), 17 de diciembre de 1961, primera página.

16 "Kennedy y Jacqueline recibidos con grandes demostraciones de aprecio", Últimas Noticias (Caracas), 17 de diciembre de 1961, p. 2.

17 "Venezuela es el país guía en Latinoamérica del Plan "Alianza para el Progreso. Dijeron senadores de EE.UU.", El Universal (Caracas), 5 de diciembre de 1961, primera página.

18 "Comienza hoy una Etapa Histórica entre EE. UU. y la América Latina", La Esfera (Caracas), 16 de diciembre de 1961, p. 9.
} 
de posesión el mandatario estadounidense ${ }^{19}$, y en una entrevista, el cardenal José Humberto Quintero, arzobispo de Caracas, expresó que el presidente Kennedy era el Jefe de Estado de una nación amiga, y además de confesión católica ${ }^{20}$. Asimismo, la Iglesia católica recibió ayuda de la Alianza para el Progreso a través del "Programa de Alimentos", a cargo de CARITAS ${ }^{21 .}$

Por otra parte, las Fuerzas Armadas siguieron con atención y participaron en el programa de la Alianza para el Progreso, a través de su principal representante, el general (FAV) Antonio Briceño Linares, ministro de Defensa, y en la figura del coronel Rafael Alfonzo Ravard, designado presidente de la Corporación Venezolana de Guayana, ente encargado de los proyectos de desarrollo en el sur amazónico. Asimismo, a lo largo de estos años las Fuerzas Armadas mostraron su apoyo a las labores de la Alianza por medio de las misiones de Acción CívicoMilitar. En especial, el Servicio de Ingeniería del Ejército realizó importantes trabajos en la construcción de vías de comunicación en varias regiones del país.

También los sindicatos de trabajadores fueron invitados a participar en la Alianza. En efecto, los directivos de la Confederación de Trabajadores de Venezuela (CTV) estuvieron en la firma de los convenios entre Betancourt y Kennedy, y en 1963 se realizó la 1a Conferencia Sindical sobre la Alianza para el Progreso en Caracas, el evento fue auspiciado por la Asociación Nacional de Empleados (ANDE), y contó con la participación de Comité Sindical para la Alianza de la CTV, en los que se discutieron los doce puntos de la Carta de Punta del Este ${ }^{22}$.

Por último, los empresarios venezolanos se sumaron optimistas a los planes de la Alianza. Organizaciones como la Unión Patronal Venezolana de Comercio habían expresado su simpatía por la visita del presidente Kennedy y sus planes de desarrollo ${ }^{23}$. Sin embargo, el mayor respaldo provino de la Asamblea Anual de la Federación de Cámaras y Asociación de Comercio y Producción de Venezuela (FEDECAMARAS), el principal organismo de privados del país, quien suscribió un documento de apoyo a la Alianza en $1962^{24}$. De este modo, los diversos sectores económicos, sociales y políticos de la nación cifraron sus esperanzas e intereses en las promesas de desarrollo de la Alianza.

\footnotetext{
19 "Discurso del presidente Kennedy al asumir la Presidencia de los EE. UU., el 20 de enero del presente año", Revista SIC, Vol. 24, No. 232, febrero 1961, pp. 88-89.

20 "Exhorta el Cardenal a los católicos para recibir a Kennedy", El Universal (Caracas), 11 de diciembre de 1961, primera página.

${ }^{21}$ Ministerio de Justicia. Memoria y Cuenta de 1965. 1965, Caracas, Imprenta Nacional, p. 134.

22 Venezuela a la Vanguardia. 1a Conferencia Sindical sobre la Alianza para el Progreso, 29 de julio - 2 de agosto 1963. 1963. Caracas, Asociación Nacional de Empelados, 34 pp.

23 "Aplaude visita de Kennedy la Unión Patronal de Comercio", Últimas Noticias (Caracas), 16 de diciembre de 1961, p. 10.

${ }^{24}$ Carta Económica de Mérida. Tesis Fundamental de los Sectores Empresariales para el Desarrollo Económico. 1962. Mérida, FEDECAMARAS. Extraído de: http://www.fedecamaras.org.ve/wp-content/uploads/2016/05/Cartaeconomica-de-Merida.pdf.
} 


\section{Actores y Planes de la Alianza para el Progreso en Venezuela}

Los presidentes Kennedy y Betancourt firmaron una declaración conjunta el 17 de diciembre de 1961, en la que se comprometieron:

“ 4 ' Los Presidentes manifestaron su determinación de lograr los objetivos de la Alianza para el Progreso, conforme a los principios del Acta de Bogotá y de la Carta de Punta del Este. Los progresos de Venezuela en la formulación y ejecución de un plan realista de largo alcance para el desarrollo económico y social, especialmente en el campo de la industria, la agricultura, la reforma agraria, la educación y la construcción de viviendas y acueductos, fueron examinados en relación con la necesidad de movilizar recursos adicionales tanto nacionales como exteriores. El Banco Interamericano de Desarrollo y el Banco Internacional de Reconstrucción y Fomento están considerando nuevos préstamos importantes además de los ya aprobados" 25 .

El documento suscrito por Kennedy y Betancourt representó la voluntad de ambos mandatarios por llevar las relaciones bilaterales e interamericanas a un nuevo nivel, a una nueva etapa. Se abre la puerta a la posibilidad de que los países latinoamericanos puedan recibir ayuda financiera para mejorar las condiciones de su población, a la vez que contrarrestar el foco de generación de resentimiento que alimenta al comunismo. Mientras que se fortalece la alianza estadounidense-venezolana en su defensa de la democracia y de la libertad en contra de violencia comunista en las Américas.

En febrero de 1963, Betancourt realizó una visita a Estados Unidos por invitación de Kennedy. Durante una rueda de prensa expresó:

"son necesarias las medidas legales de carácter defensivo contra el comunismo, como las adoptadas por el Gobierno de Venezuela, cuando el comunismo deja de ser un partido de prédica para convertirse en bandas terroristas que hacen estallar bombas y cometen asesinatos" 26 .

Las palabras de Betancourt reafirmaban la situación que se desarrollaba en Venezuela y América Latina producto de la violencia comunista; pero también recogían las posiciones objetivas que perseguía la Alianza, junto con los intereses y prioridades del Estado venezolano. De esta forma, la política exterior de Betancourt mantenía su vigencia y acuerdo con la importancia de erradicar los focos de la subversión.

\footnotetext{
25 Picón, Delia. 1999. Historia Diplomática de Venezuela. 1811-1985, Caracas, Universidad Católica Andrés Bello, p. 212.

${ }^{26}$ Betancourt, Rómulo. 2007. Antología Política. Volumen Séptimo (1959-1964), Caracas, Fundación Betancourt, p. 355.
} 
La Alianza adoptó una organización compleja al tratar satisfacer a todas las partes involucradas. De este modo, quedaron constituidos tres órganos encargados del funcionamiento de la Alianza: el Comité Tripartito OEA-BID-CEPAL, responsable de su coordinación; el Panel de Expertos, como órgano técnico especializado en la evaluación y aprobación de los planes de desarrollo nacional elaborados por los gobiernos; y la Agencia de los Estados Unidos para el Desarrollo Internacional (USAID, por sus siglas en inglés), fundada por medio de la Foreign Assistance Act de 1961, destinada a gestionar las asistencia oficial estadounidense $^{27}$.

Sin embargo, en la práctica la complejidad era mayor, puesto que participaron en distinto grado diferentes agencias estadounidenses, organismos como los Cuerpos de Paz (U.S. Peace Corps), y privados como la Rockefeller Foundation, entre otros. Por lo tanto, resulta una tarea complicada determinar con exactitud las aportaciones globales del programa en términos cuantitativos, y particulares por su misma característica.

Paralelamente, la Alianza conformó un consejo de nueve expertos, llamados los "nueve sabios", integrados por el Raúl Prebisch como coordinador, los estadounidenses Paul Rosenstein-Rodan y Harvey Perloff, el cubano Felipe Pazos, el argentino Ernesto Malaccorto, el brasileño Ari Torres, el chileno Raúl Sáez, el colombiano Hernando Agudelo Villa, el mexicano Gonzalo Robles y el guatemalteco Manuel Noriega Morales.

En el diseño de los planes y programas de la Alianza en Venezuela, tuvo un rol importante la Oficina Central de Coordinación y Planificación (CORDIPLAN), y sus directores, Dr. Manuel Pérez Guerrero (1959-1962) y Héctor Hurtado Navarro (1963-1969). CORDIPLAN había sido creada desde mediados de los cincuenta, y era el ente encargado del estudio, elaboración y evaluación de diferentes proyectos estatales destinados a generar desarrollo económico y social. Hurtado fue, además, miembro del Consejo Directivo de Instituto Latinoamericano de Planificación Económica y Social (ILPES), entre 1962 y 1965.

CORDIPLAN fue la oficina responsable de la elaboración de los planes nacionales cuatrienales, examinados anualmente. Durante los sesentas se implementaron tres planes tendientes a impulsar la industrialización del país, especialmente en el sector petrolero, hidroeléctrico y la industria minera en el sur de la nación. Así, se pusieron en marcha el I Plan de la Nación, 1960-1963, el II Plan de 1963-1966 y el III Plan de 1965-1968, todos bajo los gobiernos socialdemócratas de Betancourt y Leoni, en los que se evidencia una continuidad de los proyectos.

Asimismo, el Estado venezolano había comenzado a adelantar estudios sobre las potencialidades en recursos económicos en cada región de la nación. De modo que, durante

${ }^{27}$ Franco, Rolando. 2013. La invención del ILPES, Santiago, CEPAL, pp. 100-102. 
estos años se crearon oficinas regionales encargadas de la coordinación y planificación de las actividades de impulso al sector industrial. Así, el Estado creó la Corporación Venezolana de Guayana (CVG) en noviembre de 1960, destinada al estado Bolívar (sur amazónico); la Corporación de Desarrollo del Zulia (CORPOZULIA) en agosto de 1964, a cargo del estado Zulia, región petrolera por excelencia y con enorme potencial agrícola e industrial; la Fundación para el Desarrollo de la Región Centro-Occidental (FUDECO) en noviembre de 1964, dirigida a los estados Lara, Yaracuy y parte de Falcón; la Corporación de Desarrollo de la Región Nororiental (CORPOIENTE) en mayo de 1966, responsable de los proyectos desarrollistas en los estados Anzoátegui, Monagas, Nueva Esparta y Sucre ${ }^{28}$.

Un interesante testimonio, sobre cómo fue recibida la Alianza entre los funcionarios venezolanos, la aporta Enrique Tejera París, quien apuntó en sus memorias: "El profesor Berle no vino a hablarnos de Vietnam, sino de la Alianza para el Progreso, que concebían como un verdadero pacto multilateral (y bilateral) y no como un instrumento busca-simpatías o de dádivas" 29 .

Con la visita de Kennedy a Caracas, se realizaron varios convenios como el suscrito entre el Fondo de Préstamos y el Banco Obrero, por ejemplo. En los convenios establecidos tuvo una relevante actuación la Comisión de Ahorro y Préstamo, creada con el propósito de establecer "un nuevo sistema mutual para fomento y respaldo de ahorros de los particulares destinados a la vivienda" 30 .

La comisión estaba bajo la dirección ad honoren del señor Eugenio Mendoza Goiticoa, e integrada por Mario Mauriello, director gerente del Banco Obrero, Arturo Luis Berti, coronel Rafael Alfonzo Ravard, José Antonio Henríquez Reyes, Gonzalo Carnevali, Arturo Oria, Camilo Daza, Arturo Pardo, teniente-coronel Luis Guillermo Ferrero Tamayo, Federico Rivero Palacios, Alfredo Rodríguez Amengual, prof. Félix Poleo y Alfredo Machado Gómez.

La Comisión en sí misma era una representación de distintos sectores económicos y sociales de la vida nacional. Así, por ejemplo, se encontraban empresarios como Mendoza, uno de los más exitosos de la nación y filántropo en varias causas (Olinto, 2007); economistas como Machado Gómez, presidente del Banco Central de Venezuela (BCV, 1961-1968); educadores como Poleo, científicos como Berti, director de Malareología y Saneamiento Ambiental ${ }^{31}$, militares como Alfonzo y Ferrero, entre otros.

\footnotetext{
28 Para más información véase Pereira Iturriaga, Humberto. 1977. Las corporaciones regionales de fomento en Venezuela: una contribución al desarrollo económico, Caracas, Instituto Latinoamericano de Investigaciones Sociales - Friedrich Ebert Stiftung.

${ }^{29}$ Tejera París, Enrique. 2009. Gobierno en mano. Memorias (1958-1963), Caracas, Libros Marcados, p. 292.

${ }^{30}$ Betancourt, Rómulo. 1962. IV Mensaje Presidencial, Caracas, Oficina Central de Información, p. 81.

31 Arturo Luis Berti. Academia Nacional de Ciencias Físicas; Matemáticas y Naturales. Extraído de: http://acfiman.org/site/wp-content/uploads/2010/03/9.pdf.
} 


\section{De los planes a la práctica. La Ejecución de la Alianza para el Progreso en Venezuela}

Los planes de la Alianza se centraron en cuatro áreas primordiales: reforma agraria, educación, salud y vivienda. En cada uno de estos sectores se emprendieron proyectos para la consecución de los objetivos propuestos. De forma global, el siguiente cuadro permite observar el dinero aportado por Estados Unidos a Venezuela en forma de préstamos, donaciones y ayuda:

Cuadro No. 1. Subvenciones y Créditos Extranjeros del Gobierno Estadounidense otorgado a Venezuela de 1960 a 1970. (En millones de dólares).

\begin{tabular}{|c|c|c|c|c|c|c|c|c|c|c|c|}
\hline Programa/País & 1960 & 1961 & 1962 & 1963 & 1964 & 1965 & 1966 & 1967 & 1968 & 1969 & 1970 \\
\hline Venezuela & 6 & 32 & 64 & 43 & -40 & 40 & 41 & 48 & 33 & 33 & 16 \\
\hline
\end{tabular}

Fuente: U.S. Department of Commerce, Historical Statistics of the United States. Colonial Times to 1970. September 1975, pp. 873-875. Extraído de: https://www2.census.gov/library/publications/1975/compendia/hist_stats_colonial1970/hist_stats_colonial-1970p2-chU.pdf

El cuadro refleja cifras modestas si se le compara con otros países como Chile, Bolivia o República Dominicana, que recibieron ingresos superiores de Estados Unidos. No obstante, las condiciones de Venezuela como país exportador de petróleo le permitían compensar -y hasta cierto punto explicaba- la diferencia de recursos obtenidos de los EE.UU. en el marco de la Alianza.

En el caso de la reforma agraria, esta había comenzado en Venezuela en $1960^{32}$, antes del lanzamiento de la Alianza por Kennedy. Incluso se había tomado como modelo, el proyecto de reforma impulsado por Betancourt. No obstante, la Alianza vino a promover mucho más la reforma adelantada por Betancourt ${ }^{33}$. La administración Leoni continuó con la reforma agraria, y promulgó su reglamento ${ }^{34}$.

En educación, la Alianza permitió obtener financiamiento e impulsar la labor de varios centros de capacitación y adiestramiento en diferentes ámbitos. Así, por ejemplo, la Alianza apoyó al Instituto Nacional de Cooperación Educativa (INCE) ${ }^{35}$, destinado a la preparación técnica profesional en el área urbana para la industria, y con el Centro Interamericano de

\footnotetext{
32 Ley de Reforma Agraria. 1960. Gaceta Oficial de la República de Venezuela, No. 611 extraordinariaExtraordinaria, 19 de marzo de 1960.

33 Véase Morales Benítez, Otto. 1986. Alianza para el Progreso y Reforma Agraria, Caracas, Universidad Central de Venezuela.

34 Reglamento de la Ley de Reforma Agraria. 1967. Gaceta Oficial de la República de Venezuela, No. 1089 extraordinariaExtraordinaria, 2 de marzo de 1967.

35 La Marcha de la Alianza para el Progreso. 1961. Unión Panamericana, p. 51.
} 
Educación Rural (CIER), en Rubio, Táchira (oeste de la nación), orientado a la formación especializada en el área rural. A nivel superior, la Alianza colaboró con el Centro de Estudios de Desarrollo (CENDES) de la Universidad Central de Venezuela (UCV), dirigida a postgrados ${ }^{36}$.

En forma paralela, los Cuerpos de Paz realizaron actividades de instrucción y colaboración en la nación. Tanto en áreas urbanas como rurales, los voluntarios de estos cuerpos prestaban apoyo a labores de desarrollo. Por ejemplo, los voluntarios estadounidenses cumplían funciones de facilitadores y colaboradores en la enseñanza de oficios técnicos como carpintería o mecánica, por un período aproximado de dos años (Ver imagen No. 1). La atención prestada por la organización ha sido poco estudiada, sin embargo, el mismo director del Cuerpo de Paz, Sargent Shriver, realizó una visita al país con su equipo en $1961^{37}$.

En el campo de viviendas, la Alianza financió los proyectos habitacionales del Banco Obrero. De hecho, los propios mandatarios, Kennedy y Betancourt, fundaron el pueblo "Alianza" en el estado Carabobo ${ }^{38}$. Posteriormente se le denominó "Ciudad Alianza", como parte de una urbanización en Guacara, estado Carabobo. Otro ejemplo fue el Barrio Kennedy en Caracas, donde se construyeron 451 unidades entre apartamentos y residencias ${ }^{39}$.

En área de salud, la Alianza financió varios proyectos del Instituto Nacional de Obras Sanitarias (INOS) en el interior del país. El mismo presidente Leoni refirió en 1966, que uno de los logros de la administración había sido el combatir la malaria, la que fue prácticamente erradicada de la nación en aquella época.

Por otra parte, un aspecto interesante lo representan los diversos materiales gráficos elaborados y reproducidos por la Alianza para el Progreso, en forma de carteles aniversarios de carácter propagandístico. De esta manera, durante el segundo aniversario del programa aparecieron diversos carteles en los que se informaban los proyectos materializados por Alianza. Por ejemplo, uno de los carteles de la Alianza reportaba 8.200 salas de clases construidas $^{40}$, otro el suministro de agua potable ${ }^{41}$, entrega de 4 millones de libros ${ }^{42}$, o 160.000

\footnotetext{
${ }^{36}$ La Marcha de la Alianza para el Progreso. 1961. Unión Panamericana, p. 140.

37 "Llega Venezuela Sargent Shriver, director del Cuerpo de Paz de los Estados Unidos de América y su comitiva". ACMRE. Dirección de Protocolo, Estados Unidos, 1961, Exp. No. 710, DP.1.st. Memoria del Ministerio de Relaciones Exteriores de Venezuela, 1961, pp. LXXII.

38 "Fundación del pueblo Alianza, Venezuela recibe un préstamo por doce millones de dólares", La Esfera (Caracas), 17 de diciembre de 1961.

${ }^{39}$ Documentos Presidenciales. Tomo IV. 1968, p. 6.

40 Biblioteca Nacional de Venezuela (BNV). "Escuelas hemos construido 8200 aulas en dos años". Alianza para el Progreso. 2o Aniversario. Cartel (74 x $56 \mathrm{~cm})$, [1963].

${ }^{41}$ BNV. "Más agua potable hemos instalado". Alianza para el Progreso. 2을 Aniversario. Cartel $(74 \times 56 \mathrm{~cm})$, [1963].

42 BNV. "Hemos distribuido 4 millones de libros de texto". Alianza para el Progreso. 20 Aniversario. Cartel (74 x 56 cm), [1963].
} 
préstamos agrícolas ${ }^{43}$, entre otros. Estas representaciones visuales, además de tener un objetivo divulgativo, también expresaban el carácter del programa para proyectarse hacia distintos sectores sociales y por diferentes medios.

De forma paralela, la Alianza y las relaciones bilaterales con los Estados Unidos marchaban de la mano. Así, los encuentros entre las dos naciones pasaban no solo los puntos tradicionales de la agenda, petróleo y comercio, sino también por los lazos en común, promoción de la democracia en la región, lucha contra la subversión comunista y la Alianza. Estos encuentros se realizaban periódicamente en varios niveles, tanto de presidentes y cancilleres ${ }^{44}$ como de embajadores y altos funcionarios.

\section{Influencia de la Alianza en Venezuela}

El año 1963 fue crucial para la Alianza, no solo por la desaparición física del presidente Kennedy sino por lo que este significaría en la marcha del proyecto. Si bien el nuevo mandatario estadounidense, Lyndon B. Johnson, se comprometió a continuar la política de la Alianza hacia América Latina, esta comenzó a cambiar de matices en los sucesivos años de 1964 a 1966.

Por su parte, el nuevo presidente venezolano Raúl Leoni asumió el cargo en marzo de 1964, y desde un primer momento manifestó su compromiso por conservar la doctrina Betancourt como lineamiento de su política exterior y la intención de continuar las mejorar relaciones con los Estados Unidos ${ }^{45}$.

A fines de noviembre de 1965, el senador Robert "Bobby" Kennedy visitó Caracas, y declaró: "cuando el presidente Kennedy estructuró la Alianza para el Progreso pensó en Venezuela como piedra angular"46. Las palabras de Bobby Kennedy aportan una idea de la atmósfera que se vivía en aquel momento, no solo entorno a la memoria de su hermano sino también de la Alianza y lo que representaba Venezuela como socio.

Sin embargo, dos hechos enturbiarían el camino de la Alianza; la guerra de Vietnam y la crisis dominicana en 1965. La primera, supondría la prioridad para la administración Johnson, y por consiguiente absorbería recursos estadounidenses; la segunda en tanto, dejó un sabor amargo

\footnotetext{
${ }^{43}$ BNV. “Con 160.00 préstamos agrícolas en dos años hemos aumentado la producción”. Alianza para el Progreso. Cartel $(102 \times 77 \mathrm{~cm})$, [1963].

44 Las visitas del canciller venezolano a Kennedy en 1963, y la visita del secretario de Estado estadounidense a Leoni en 1965. "Falcón Briceño se entrevistó con Kennedy", El Nacional (Caracas), 3 de agosto de 1963. U.S. Department of State Archive. Dean Rusk (January 21, 1961 - January 20, 1969). Extraído de: https://20012009.state.gov/r/pa/ho/trvl/ls/13034.htm.

45 Romero, María Teresa. 2009. Política Exterior venezolana: el proyecto democrático, 1959-1999. Caracas, Libros El Nacional, p. 49.

46 "Kennedy alborotó los barrios y hubo gritos y aplausos en manifestación popular", El Nacional (Caracas), 1ㅇde diciembre de 1965, p. D-10.
} 
en las relaciones interamericanas, al mostrarse una actitud agresiva de Johnson, que preocupó a los gobiernos de Leoni y Eduardo Frei ${ }^{47}$.

No obstante, las administraciones de Washington y Caracas entendían que eran aliados con intereses comunes en la región, lo que abrió las puertas para mantener encuentros. Así, en agosto de 1966, Johnson y Leoni sostuvieron una conversación telefónica a través del nuevo cable submarino entre ambas naciones, en la que Johnson señaló:

"We have added a new dimension to our endeavors now by undertaking the work of the Alliance for Progress.

In all of these enterprises we share an abiding concern for the freedom and the dignity of man, and our number one objective in the world is peace for all mankind.

So now as this cable opens a new era of improved communications between our countries, we can look forward to even closer friendships and associations between Americans and Venezuelans" ${ }^{\prime \prime}$.

Quedaba claro, Johnson seguía proyectando la Alianza para el Progreso como marco de referencia y parte importante de los valores y principios compartidos en las relaciones venezolano-estadounidenses, en las que se enfatizaban aspectos del soft power como las coincidencias ideológicas en la libertad, la dignidad del hombre y la amistad entre ambas naciones. Asimismo, en medio de la Conferencia de Punta del Este, en Uruguay, el 11 de abril de 1967, se reunieron los presidentes Johnson y Leoni. Durante el encuentro, Leoni mostró su preocupación por el precio del petróleo venezolano en los Estados Unidos, y sostuvo:

"Venezuela is a nation that is building a democracy with strong foundations. Its policy has reflected the principles that were endorsed in this very place (Punta del Este) at the time the Alliance for Progress was launched a few years ago. Venezuela has brought about a transformation in the lives of its people in the rural as well as in the urban areas. This has involved considerable expenditure on the part of the Venezuelan Government. This, of course, is something that the Venezuelan Government desires, and a part of this is the wish to promote industry in the country. But Venezuela in the past has been fertile soil to the natural enemies of democracy in Venezuela, and of the United States" ${ }^{49}$.

\footnotetext{
${ }^{47}$ Fermandois, Joaquín .2004, Mundo y fin de mundo. Chile en la política mundial 1900-2004, Santiago, Pontificia Universidad Católica de Chile, p. 306.

48366 - Remarks by Telephone with Presidente Leoni of Venezuela Inaugurating a Cubmarine Cable Linking the Two Countries. August 3, 1966". Extraído de: http://www.presidency.ucsb.edu/ws/index.php?pid=27755.

49 Memorandum of Conversation. Punta del Este, Uruguay, April 11, 1967, 6 p.m. Extraído de: https://history.state.gov/historicaldocuments/frus1964-68v31/d540.
} 
De este modo, Leoni renueva sus votos por los compromisos de la Alianza, pero devela también los intereses más pragmáticos que mantiene Venezuela en relación con su principal comprador de petróleo. Sin lugar a dudas, los actores políticos conductores en la práctica de la política exterior y de la Alianza habían cambiado, y se hacía notoria la diferencia de la otrora relación Kennedy-Betancourt.

Pese a que Venezuela pudo fortalecer sus instituciones democráticas y derrotar militarmente a las guerrillas comunistas, con el apoyo estadounidense a la novel democracia suramericana, ya para 1968 se hacía evidente una penumbra en el ánimo en torno a la Alianza para el Progreso. Leoni consideraba que la Alianza no había podido responder a los objetivos y esperanzas cifradas en ella. En su opinión, dos razones perjudicaron su desarrollo:

"De un lado, los poderosos grupos económicos, sordos a la implantación de medidas que beneficien a las grandes mayorías sociales, ajenos al proceso históricos y social que conmueve al mundo. Y del otro, grupos minoritarios, agentes foráneos de la subversión comunistas, interesados en socavar las instituciones democráticas, los cuales actúan como agentes de perturbación en el normal desarrollo de nuestras sociedades.

A esto se añade que muchas veces los esfuerzos internos se vieron entrabados por la tardía o complicada ayuda financiera externa, que nunca llega en el momento oportuno" ${ }^{50}$.

Las reflexiones y críticas de Leoni reflejaban, en buena medida, la consternación por las razones endógenas y exógenas que sopesaron el incumplimiento de las expectativas de la Alianza. Los obstáculos que tuvo que enfrentar la Alianza fueron diversos. Por una parte, problemas estructurales de las economías locales, la dependencia a la exportación de materias primas, además de la violencia comunista. Mientras que, por otra parte, los mecanismos de financiamiento de los proyectos de la Alianza presentaron problemas de gestión, lo que deja en el panorama responsabilidades compartidas entre latinoamericanos y estadounidenses.

\section{Consideraciones finales}

La Alianza para el Progreso no cambió las estructuras de desarrollo económico, esto debido a problemas mucho más estructurales que temporales. Pero sí representó un importante apoyo político y diplomático para la democracia venezolana en momentos en que atravesaba distintos y fuertes ataques ${ }^{51}$. Por un lado, la agresión externa de la Cuba comunista, y por otro,

\footnotetext{
50 Leoni, Raúl. 1969. Documentos Presidenciales. Tomo IV, Caracas: Imprenta Nacional, p. 282

51 La relación de Estados Unidos con las democracias en América Latina ha sido objeto de intensos debates hasta el día de hoy. En algunos casos, se ha señalado el rol de Estados Unidos en el reconocimiento de gobiernos surgidos de golpes de Estado, como en Argentina en 1962 o Brasil en 1964. En otros casos, se ha apuntado el papel de Cuba en la promoción de guerrillas para desestabilizar gobiernos en América Latina, como en Bolivia en 1966, o de
} 
internamente enfrentaba la violencia de la guerrilla comunista FALN, y dos intentos de golpes de Estado en 1962.

Particularmente, el caso de la Alianza para el Progreso en Venezuela representó una experiencia de pragmatismo y realismo político (tomando la concepción de Hans Morgenthau), al establecerse un acuerdo estratégico. Por un lado, Venezuela y Betancourt se convirtieron en modelos regionales de un gobierno civil y democrático, apoyado y promocionado por Estado Unidos. Por otro lado, Betancourt y Venezuela necesitaban de las condiciones de alianza política y seguridad hemisférica para fortalecer sus jóvenes institucionalidad democrática (desde 1958) y poder vencer la violencia de las guerrillas comunistas.

La relación Betancourt-Kennedy fue fundamental para las relaciones bilaterales y el apoyo a la Alianza. Por una parte, Betancourt eran considerado un líder intelectual y político en América Latina, perfil ganado por sus años de lucha las dictaduras militares de Pérez Jiménez en Venezuela y de Trujillo en la República Dominicana; y, además, un hombre que había recorrido varios países en sus años de exilio y publicado investigaciones sobre los problemas venezolanos y latinoamericanos.

Asimismo, Betancourt era un firme anticomunista, como quedó plasmado en su famosa Doctrina Betancourt: "se debe extender un cordón sanitario sobre los regímenes de facto, militares y comunistas". Por su parte, Kennedy se mostraba entusiasta por lo que podía hacer en la región, admiraba la determinación de Betancourt y estimuló su proyección como socio y aliado en la región.

Sin embargo, la relación Leoni-Johnson fue distinta. Leoni era un hombre más reservado que Betancourt, mientras que Johnson tenía su interés puesto en la Guerra de Vietnam y en los propios problemas estadounidenses entorno a los derechos civiles. Además, la crisis dominicana de 1965 y el envío de tropas norteamericanas distanciaron, aunque brevemente, las posiciones de ambos. Leoni se mostró contrario a la intervención unilateral de Johnson.

Por último, como una arista investigativa, la presencia y labor de los Cuerpos de Paz en Venezuela es uno de los temas interesantes a resolver, y amerita una indagación más exhaustiva en fuentes primarias para determinar su alcance e influencia.

reconocer administraciones fruto de golpes de Estado como en Perú en 1969, cuyas relaciones de restablecieron en 1972. VéasePuede verse: Schoultz, Lars. 1998. Beneth the United States: A History of U.S. Policy toward Latin America, Cambridge, MA, Harvard University Press; O'Brien, Thomas. F. 2007. Making the Americas. The United States and Latin America, from the Age of Revolutions to the Era Globalization. Albuquerque, University of New Mexico Press; Gay-Sylvestre, Dominique. (Ed.). 2007. La Revolución Cubana. Miradas cruzadas (1959-2006), Tenerife, España, Ediciones Idea; Pettiná, Vanni. 2018. Historia mínima de la Guerra fría en América Latina, México, DF, EL Colegio de México; Palacios, Marco. (Dir.). 2008. Historia general de América Latina, España, UNESCO - Editorial Trotta (Volumen VIII); Rojas, Rafael. 2015. Historia mínima de la Revolución Cubana, México, DF, El Colegio de México, entre otros. 


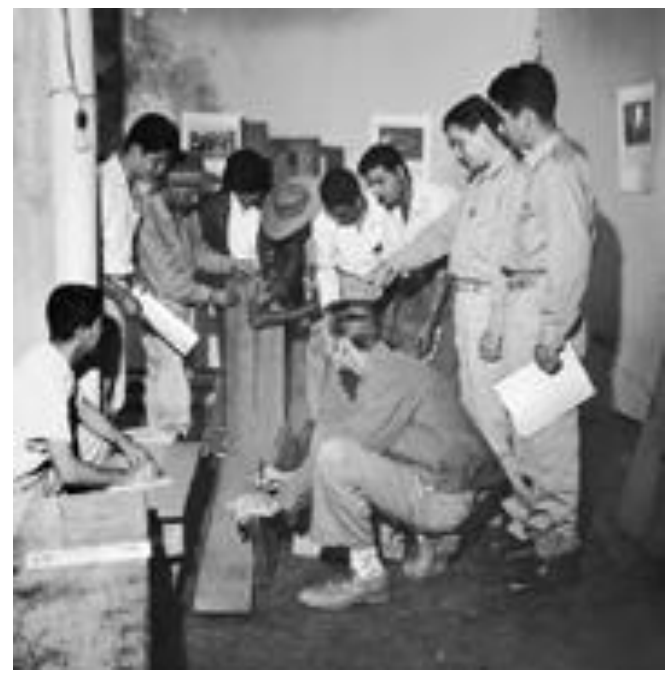

Imagen No. 1. Miembro de Cuerpo de Paz estadounidense enseñando a trabajar carpintería a vecinos de Boconó, estado Trujillo, 1963. Fuente: Colección Shell. Repositorio Institucional de la Universidad Católica Andrés Bello (Caracas) ${ }^{52}$.

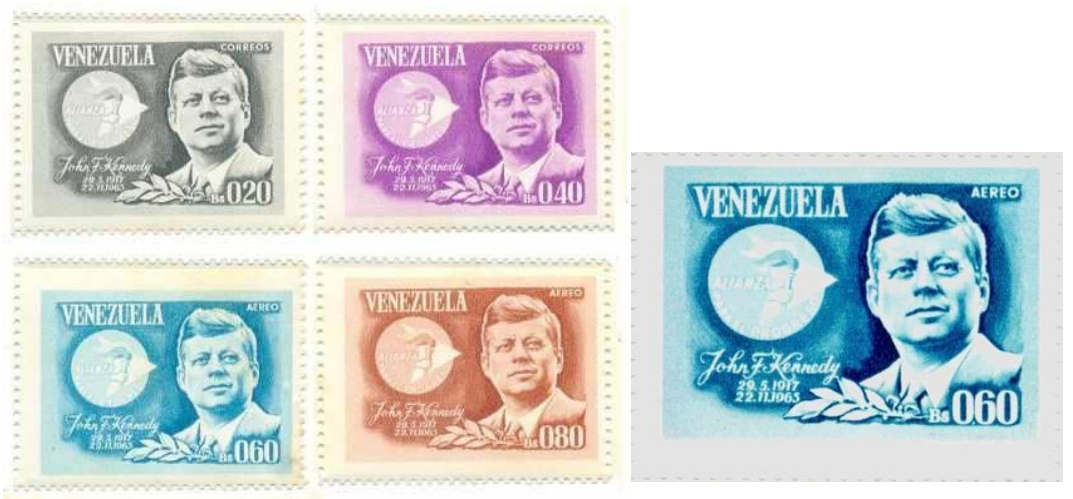

Imagen No. 2. Timbres de correo postal emitidas por Venezuela en 1965, con motivo de la Alianza para el Progreso. Gaceta Oficial de la República de Venezuela, No. 27.456, del 03 de junio de 1964.

\section{Bibliografía}

Alianza para el Progreso. 1961. Documentos Básicos, Punta del Este, s.e.

Betancourt, Rómulo. 2007. Antología Política. Volumen Séptimo (1959-1964), Caracas, Fundación Betancourt.

Betancourt, Rómulo. 1962. IV Mensaje Presidencial, Caracas, Oficina Central de Información.

Betancourt, Rómulo. 1962. Tres años de gobierno democrático, 1959-1962, Caracas, Imprenta Nacional.

52 Extraído de: http://saber.ucab.edu.ve/handle/123456789/16847 
Biblioteca Nacional de Venezuela (BNV). 1963. Alianza para el Progreso. 2ㅇ Aniversario.

Buttó, Luis Alberto. 2015. "El golpismo se viste de blanco. Insurrecciones navales contra la naciente democracia venezolana", en Tiempo y Espacio, No. 64, Caracas, pp. 509-544.

FEDECAMARAS. 1962. Carta Económica de Mérida. Tesis Fundamental de los Sectores Empresariales para el Desarrollo Económico, Mérida, extraído de: http://www.fedecamaras.org.ve/wpcontent/uploads/2016/05/Carta-economica-de-Merida.pdf

Castillo D'Imperio, Ocarina. 2003. Los años del Buldozer: ideología y política, 1948-1958, Caracas, Tropykos.

CORDIPLAN. 1960. I Plan de la Nación 1960-1964, Caracas, s.e.

CORDIPLAN. 1963. II Plan de la Nación 1963-1966, Caracas, s.e.

CORDIPLAN. 1965. III Plan de la Nación 1965-1968, Caracas, s.e.

Fermandois, Joaquín. 2004. Mundo y fin de mundo. Chile en la política mundial 1900-2004, Santiago, UC. Franco, Rolando. 2013. La invención del ILPES, Santiago, CEPAL.

Gay-Sylvestre, Dominique. (Ed.). 2007. La Revolución Cubana. Miradas cruzadas (1959-2006), Tenerife, España, Ediciones Idea.

Guzmán, Guillermo. 2009. “La Oficina de Estudios Especiales de la Presidencia de la República: la planificación de la Industria Siderúrgica Nacional y el desarrollo de Guayana (1953-1958)", Tiempo y Espacio, Vol. 19, No. 52, Caracas, pp. 251-268.

Irwin Gáffaro, Domingo. 2006. Control Civil y Pretorianismo en Venezuela, Caracas, Universidad Católica Andrés Bello.

Johnson, Paul. 2000. Tiempos Modernos, Barcelona, Vergara.

Kennedy, John. 1961. "Discurso del presidente Kennedy al asumir la Presidencia de los EE. UU., el 20 de enero del presente año", en Revista SIC, Vol. 24, No. 232, Caracas, febrero 1961, pp. 88-89.

Unión Panamericana. 1961. La Marcha de la Alianza para el Progreso. Washington DC.

Leoni, Raúl. 1969. Documentos Presidenciales, Caracas, Imprenta Nacional, Tomo IV.

Estado Venezolano. 1960. "Ley de Reforma Agraria”, en Gaceta Oficial de la República de Venezuela, No. 611 extraordinaria, 19 de marzo de 1960, Caracas, Estado Venezolano.

Estado Venezolano. 1967. "Reglamento de la Ley de Reforma Agraria”, en Gaceta Oficial de la República de Venezuela, No. 1089 extraordinaria, 2 de marzo de 1967, Caracas, Estado Venezolano.

López Portillo, Felícitas. 1986. El Perezjimenismo: génesis de las dictaduras desarrollistas, México, UNAM. Mayobre, José Antonio. 2007. Latinoamérica: Economía y desarrollo, Caracas, Banco Central de Venezuela.

Memorandum of Conversation. Punta del Este, Uruguay, April 11, 1967, 6 p.m. Extraído de: https://history.state.gov/historicaldocuments/frus1964-68v31/d540.

Miller, Aragorn Storm. 2016. Precarious Paths to Freedom: The United States, Venezuela, and the Latin American Cold War, Albuquerque, University of New Mexico Press.

Ministerio de Justicia. 1965. Memoria y Cuenta de 1965, Caracas, Imprenta Nacional.

Ministerio de Relaciones Exteriores de Venezuela. 1962. Memoria del Ministerio de Relaciones Exteriores de Venezuela 1961, Caracas, Imprenta Nacional.

Morales Benítez, Otto. 1986. Alianza para el Progreso y Reforma Agraria, Caracas, Universidad Central de Venezuela.

Olinto, Oscar. 2007. Eugenio Mendoza. Empresario social de la vivienda popular en Venezuela, Caracas, Fondo Editorial Vivienda Popular.

O'Brien, Thomas. F. 2007. Making the Americas. The United States and Latin America, from the Age of Revolutions to the Era Globalization, Albuquerque, University of New Mexico Press. 
Palacios, Marco (Dir.). 2008. Historia General de América Latina, España, UNESCO - Editorial Trotta (Volumen VIII).

Pereira Iturriaga, Humberto. 1977. Las corporaciones regionales de fomento en Venezuela: una contribución al desarrollo económico, Caracas, Instituto Latinoamericano de Investigaciones Sociales Friedrich Ebert Stiftung.

Pettiná, Vanni. 2018. Historia mínima de la Guerra Fría en América Latina, México DF, EL Colegio de México.

Picón, Delia. 1999. Historia Diplomática de Venezuela. 1811-1985, Caracas, Universidad Católica Andrés Bello.

Rabe, Stephen. 1999. The Most Dangerous Area in the World: John F. Kennedy Confronts Communist Revolution in Latin America, Chapel Hill, University of North Carolina Press.

Ramos Rodríguez, Froilán. 2013. “Venezuela 1936-1945. ¿Democracia bajo tutelaje militar o Transición pretoriana?", en Cardozo, Alejandro y Buttó, Luis Alberto (Dir.). El Incesto Republicano. Relaciones Civiles y Militares en Venezuela 1812-2012, Caracas, Nuevos Aires, pp. 101-131.

Ramos Rodríguez, Froilán y Buttó, Luis Alberto. 2015. "El golpismo se viste de blanco. Insurrecciones navales contra la naciente democracia venezolana”, en Tiempo y Espacio, No. 64, Caracas, pp. 509544.

Ramos Rodríguez, Froilán y Caripe, Germán. 2013. “Proyecto de creación una Industria Básica de apoyo a la Fuerza Armada de Venezuela durante el Gobierno militar de Marcos Pérez Jiménez (1952-1958)", RUHM, Vol. 2, No. 3, Madrid, pp. 203-218.

Ramos Rodríguez, Froilán y Javier Castro A. 2014. "La Alianza para el Progreso en Chile y Venezuela, 19611963", en Tiempo y Espacio, No. 62, Caracas, pp. 99-138.

Rojas, Rafael. 2015. Historia mínima de la Revolución Cubana, México, DF, El Colegio de México.

Romero, María Teresa. 2009. Política Exterior venezolana: el proyecto democrático, 1959-1999, Caracas, Libros El Nacional.

Romero, María Teresa. 2005. Venezuela en defensa de la democracia, 1958-1998. El caso de la Doctrina Betancourt, Caracas, Fondo para la Cultura Urbana.

Schoultz, Lars. 1998. Beneth the United States: A History of U.S. Policy toward Latin America, Cambridge, MA, Harvard University Press.

Taffet, Jeffrey F. 2007. Foreign Aid as Foreign Policy: The Alliance for Progress in Latin America, New York, Routledge.

Tarver, H. Michael. 2001. Venezuelan Insurgency, 1960-1968: A successful failure, Bloomington, Indiana, Xlibris.

Tejera París, Enrique. 2009. Gobierno en mano. Memorias (1958-1963), Caracas, Libros Marcados.

The American Presidency Project. 1966. 366 -Remarks by Telephone with Presidente Leoni of Venezuela Inaugurating a Cubmarine Cable Linking the Two Countries. August 3, 1966. Extraído de: http://www.presidency.ucsb.edu/ws/index.php?pid=27755

U.S. Department of State Archive. Dean Rusk (January 21, 1961 - January 20, 1969). Extraído de: https://2001-2009.state.gov/r/pa/ho/trvl/ls/13034.htm

Wright, Thomas. 2001. Latin America in the Era of Cuban Revolution, New York, Praeger.

Asociación Nacional de Empleados. 1963. Venezuela a la Vanguardia. 19 Conferencia Sindical sobre la Alianza para el Progreso, 29 de julio - 2 de agosto 1963, Caracas, Asociación Nacional de Empelados, 1963, s/e.

"Venezuela's President Betancourt", Times, Feb. 8, 1960. 
Hemerografía:

El Nacional, 1961-1969 (Caracas)

El Universal, 1961-1969 (Caracas)

La Esfera, 1961-1969 (Caracas)

Últimas Noticias, 1961-1969 (Caracas) 